\title{
Dark matter spin characterization in mono- $Z$ channels
}

\author{
W. Abdallahø, ${ }^{1,2, *}$ A. Hammad, ${ }^{3, \dagger}$ S. Khalil, ${ }^{4, *}$ and S. Moretti, ${ }^{5, \pi}$ \\ ${ }^{1}$ Harish-Chandra Research Institute, Chhatnag Road, Jhunsi, Allahabad 211019, India \\ ${ }^{2}$ Department of Mathematics, Faculty of Science, Cairo University, Giza 12613, Egypt \\ ${ }^{3}$ Department of Physics, University of Basel, Klingelbergstraße 82, CH-4056 Basel, Switzerland \\ ${ }^{4}$ Center for Fundamental Physics, Zewail City of Science and Technology, \\ 6 October City, Giza 12588, Egypt \\ ${ }^{5}$ School of Physics and Astronomy, University of Southampton, \\ Highfield, Southampton SO17 1BJ, United Kingdom
}

(Received 6 August 2019; published 8 November 2019)

\begin{abstract}
The $B-L$ supersymmetric Standard Model (BLSSM) is an ideal testing ground of the spin nature of dark matter (DM) as it offers amongst its candidates both a spin- $1 / 2$ (the lightest neutralino) and spin- 0 (the lightest right-handed sneutrino) state. We show that the mono- $Z$ channel can be used at the Large Hadron Collider (LHC) to diagnose whether a DM signal is characterized within the BLSSM by a fermionic or (pseudo)scalar DM particle. Sensitivity to either hypothesis can be obtained after only $100 \mathrm{fb}^{-1}$ of luminosity following runs 2 and 3 of the LHC.
\end{abstract}

DOI: 10.1103/PhysRevD.100.095006

\section{INTRODUCTION}

Dark matter (DM) is one of the firm pieces of evidence of physics beyond the Standard Model (BSM). Searches for DM at the Large Hadron Collider (LHC) through missing transverse energy (MET or $E_{T}$ ) while probing a single particle, like monojet, monophoton, mono- $Z$, and monoHiggs, are one of the most promising methods for establishing DM existence directly in an experiment. However, the nature of DM remains one of the foremost open questions in particle physics, especially whether the DM is a fermionic or bosonic particle.

Fermionic DM is predicted by several BSMs, like the minimal supersymmetric Standard Model (MSSM), in which the lightest neutralino (a fermionic superpartner of the neutral scalar and gauge bosons of the SM) is a quite popular example of weak scale DM. Scalar DM has been analyzed in models with extra inert singlet or doublet Higgs bosons. Here, we will perform a comparative study for the two types of DM, predicted by the same model, the $B-L$ supersymmetric Standard Model (BLSSM), in different regions of parameter space.

\footnotetext{
*waleedabdallah@hri.res.in

†ahmed.hammad@unibas.ch

\$skhalil@zewailcity.edu.eg

is.moretti@soton.ac.uk
}

Published by the American Physical Society under the terms of the Creative Commons Attribution 4.0 International license. Further distribution of this work must maintain attribution to the author(s) and the published article's title, journal citation, and DOI. Funded by SCOAP ${ }^{3}$.
The BLSSM is a natural extension of the MSSM with an extra $U(1)_{B-L}$. It accounts for nonvanishing neutrino masses through a low scale seesaw mechanism, which can be an inverse seesaw (see Ref. [1] for a review). In this scenario, it is quite possible to have the lightest neutralino or the lightest right-handed sneutrino as the lightest supersymmetric particle (LSP), so that any of these can be a stable DM candidate [2]. A detailed analysis of BLSSM DM candidates has been performed in [2,3] (see also [4]). Therein, it was shown that, for a wide region of parameter space, the lightest right-handed sneutrino, with mass of order $\mathcal{O}(100) \mathrm{GeV}$, can be a viable DM candidate that satisfies the limits of relic abundance and also the scattering cross sections with nuclei. The chances of the lightest neutralino being the actual DM state are much less in comparison; however, in some regions of the parameter space, it is still possible to have it as the origin of DM, in particular, in the form of the lightest $B-L$ neutralino. Further, in Ref. [3], it was shown that the Fermi Large Area Telescope (FermiLAT) can be sensitive to the DM spin (and eventually distinguish between the sneutrino and neutralino hypotheses) in the study of high-energy $\gamma$-ray spectra emitted from DM (co)annihilation into $W^{ \pm}$boson pairs (in turn emitting photons).

Furthermore, we studied several single-particle signatures of the BLSSM DM at the LHC, i.e., monojet, monophoton, mono- $Z$, and mono-Higgs signals, induced by new channels mediated by the heavy $Z^{\prime}$ (in the few $\mathrm{TeV}$ range) pertaining to the (broken) $U(1)_{B-L}$ group $[5,6]$. The salient feature of this BLSSM specific channel is that the final state monoprobe carries a very large MET. Hence, it is 
a clean signal, almost free from SM background. It was argued that, with luminosities of order $100 \mathrm{fb}^{-1}$, monojet events associated with BLSSM DM can be accessible at the LHC while monophoton, mono- $Z$, and mono-Higgs signals can be used as diagnostic tools of the underlying scenario.

In this paper, we expand on all these results, by showing that DM spin can be accessed at the LHC in the mono- $Z$ channel. We prove this result by showing that the angular distributions of the final state lepton emerging from a subsequent $Z$ decay, for both neutralino and right-handed sneutrino DM, are significantly different from each other. This is in contrast to the result that these distributions are identical in monojet, monophoton, and mono-Higgs (owing to the fact that jets and $\gamma$ 's do not couple directly to DM while Higgs radiation is isotropic), thus being insensitive to the DM spin.

This paper is organized as follows. In Sec. II we briefly highlight the possibility of having both (pseudo)scalar and fermionic DM in the BLSSM with an inverse seesaw mechanism. Section III is dedicated to the mono- $Z$ analysis in these two DM scenarios. In Sec. IV we discuss the impact of the DM spin on the angular distributions of the corresponding final leptons. Our conclusions and final remarks are given in Sec. V.

\section{SCALAR VERSUS FERMIONIC DM}

The BLSSM is based on the gauge group $S U(3)_{C} \times$ $S U(2)_{L} \times U(1)_{Y} \times U(1)_{B-L}$, where the $U(1)_{B-L}$ is spontaneously broken at the $\mathrm{TeV}$ scale [7] by chiral singlet superfields $\hat{\eta}_{1,2}$ with $B-L$ charge $= \pm 1$. Here, a gauge boson $Z^{\prime}$ and three chiral singlet superfields $\hat{\nu}_{i}$ with $B-L$ charge $=-1$ are introduced for the consistency of the model. Finally, three chiral singlet superfields $\hat{S}_{1}$ with $B-L$ charge $=+2$ and three chiral singlet superfields $\hat{S}_{2}$ with $B-L$ charge $=-2$ are considered to implement the inverse seesaw mechanism [8]. The superpotential is given by

$$
\begin{aligned}
W= & Y_{u} \hat{Q} \hat{H}_{2} \hat{U}^{c}+Y_{d} \hat{Q} \hat{H}_{1} \hat{D}^{c}+Y_{e} \hat{L} \hat{H}_{1} \hat{E}^{c} \\
& +Y_{\nu} \hat{L} \hat{H}_{2} \hat{\nu}^{c}+Y_{S} \hat{\nu}^{c} \hat{\eta}_{1} \hat{S}_{2}+\mu \hat{H}_{1} \hat{H}_{2}+\mu^{\prime} \hat{\eta}_{1} \hat{\eta}_{2} .
\end{aligned}
$$

The neutralinos, $\tilde{\chi}_{i}^{0}(i=1, \ldots, 7)$, are the physical (mass) superpositions of the three fermionic partners of the neutral gauge bosons, called gauginos, of the neutral MSSM Higgs bosons $\left(\tilde{H}_{1}^{0}\right.$ and $\left.\tilde{H}_{2}^{0}\right)$, called Higgsinos, and of the $B-L$ scalar bosons $\left(\tilde{\eta}_{1}\right.$ and $\left.\tilde{\eta}_{2}\right)$. In this regard, the lightest neutralino, in the basis $\psi^{0}=\left\{\tilde{B}, \tilde{W}^{3}, \tilde{H}_{1}^{0}, \tilde{H}_{2}^{0}, \tilde{B}^{\prime}, \tilde{\eta}_{1}, \tilde{\eta}_{2}\right\}$, decomposes as

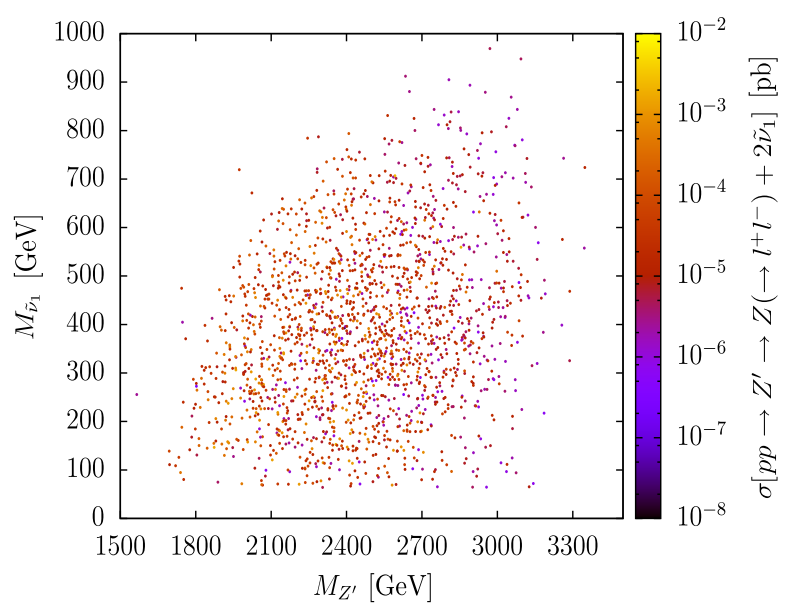

FIG. 1. The cross section for $p p \rightarrow Z^{\prime} \rightarrow Z\left(\rightarrow l^{+} l^{-}\right)+2 \tilde{\nu}_{1}$ at the LHC with $\sqrt{s}=14 \mathrm{TeV}$ mapped over the $Z^{\prime}$ and $\tilde{\nu}_{1}$ masses for the BLSSM with an inverse seesaw mechanism.

$$
\tilde{\chi}_{1}^{0}=\sum_{i=1}^{7} V_{1 i} \psi_{i}^{0} .
$$

The lightest sneutrino $\tilde{\nu}_{1}$ (either a $C P$-even state, $\tilde{\nu}_{1}^{\mathrm{R}}$, or a $C P$-odd one, $\tilde{\nu}_{1}^{\mathrm{I}}$ ) can be expressed in terms of $\tilde{\nu}_{L}^{+}, \tilde{\nu}_{R}^{+}$, and $\tilde{S}_{2}^{+}$ (e.g., in the case of it being $C P$ even) as

$\tilde{\nu}_{1}=\sum_{i=1}^{3} R_{1 i}\left(\tilde{\nu}_{L}^{+}\right)_{i}+\sum_{j=1}^{3} R_{1 j}\left(\tilde{\nu}_{R}^{+}\right)_{j}+\sum_{k=1}^{3} R_{1 k}\left(\tilde{S}_{2}^{+}\right)_{k}$,

where $R_{1 i} \approx\{0,0,0\}, \quad R_{1 j}=\frac{1}{\sqrt{2}}\{1,0,0\}, \quad$ and $\quad R_{1 k}=$ $\frac{1}{\sqrt{2}}\{1,0,0\}$, which confirms that the lightest sneutrino is mainly right handed (i.e., a combination of $\tilde{\nu}_{R}^{+}$and $\tilde{S}_{2}^{+}$).

It is worth mentioning that, due to the $U(1)_{Y}$ and $U(1)_{B-L}$ gauge kinetic mixing, the mass of the extra neutral gauge boson, $Z^{\prime}$, is given by

$$
M_{Z^{\prime}}^{2}=g_{B-L}^{2} v^{\prime 2}+\frac{1}{4} \tilde{g}^{2} v^{2},
$$

where $\tilde{g}$ is the gauge kinetic mixing coupling. Also, the mixing angle between $Z$ and $Z^{\prime}$, which is experimentally limited to $\lesssim \mathcal{O}\left(10^{-3}\right)$, is given by

$$
\tan 2 \theta^{\prime}=\frac{2 \tilde{g} \sqrt{g_{1}^{2}+g_{2}^{2}}}{\tilde{g}^{2}+4\left(\frac{v^{\prime}}{v}\right)^{2} g_{B-L}^{2}-g_{2}^{2}-g_{1}^{2}} .
$$

The relevant interactions of the lightest neutralino and lightest right-handed sneutrino with the $Z^{\prime}$ and $Z$ bosons are given by

$$
\begin{aligned}
\mathcal{L}_{\mathrm{int}} \simeq & -i\left(\frac{\tilde{g}}{2} \Delta V_{34}+g_{B-L} \Delta V_{67}\right) \overline{\tilde{\chi}_{1}^{0}} Z^{\prime} \gamma_{5} \tilde{\chi}_{i}^{0}+\frac{g_{B-L}}{2} \sum_{n=1}^{3} \tilde{\nu}_{1}^{\mathrm{R}} \tilde{\nu}_{j}^{\mathrm{I}}\left(p^{\prime}-p\right)^{\mu} Z_{\mu}^{\prime}\left(I_{j, n+6}^{*} R_{1, n+6}^{*}-I_{j, n+3}^{*} R_{1, n+3}^{*}\right) \\
& -\frac{i}{2}\left[\left(g_{2} \cos \theta_{W}+g_{1} \sin \theta_{W}\right) \Delta V_{34}-2 \tilde{g} \Delta V_{67}\right] \overline{\tilde{\chi}_{1}^{0}} Z \gamma_{5} \tilde{\chi}_{i}^{0}+\frac{1}{2}\left(g_{2} \cos \theta_{W}+g_{1} \sin \theta_{W}\right) \sum_{n=1}^{3} \tilde{\nu}_{1}^{\mathrm{R}} \tilde{\nu}_{j}^{\mathrm{I}}\left(p^{\prime}-p\right)^{\mu} Z_{\mu} I_{j, n}^{*} R_{1, n}^{*},
\end{aligned}
$$



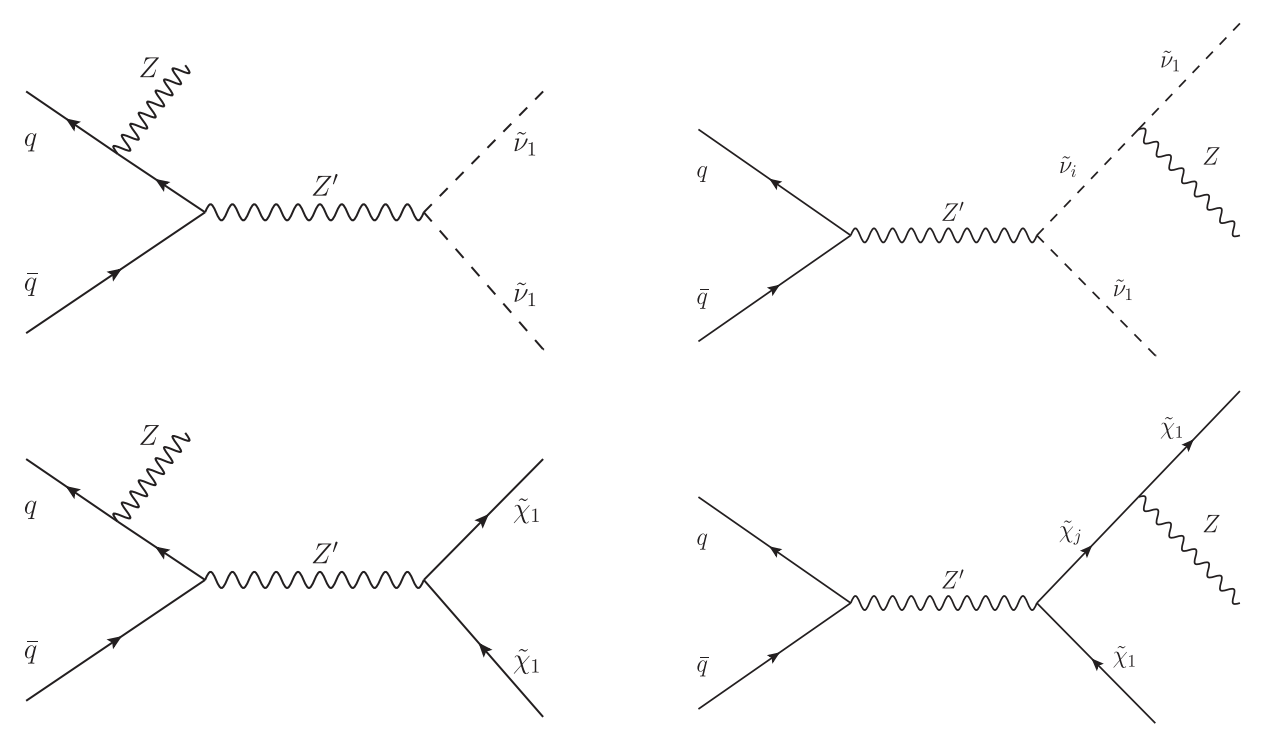

FIG. 2. Feynman diagrams for mono- $Z$ signals of scalar (top panels) or fermionic (bottom panels) DM: $S_{1}$ (left) and $S_{2}$ (right), corresponding to $Z$ initial state radiation (ISR) and final state radiation (FSR), respectively. Here, $i=2,3, \ldots, 9$ and $j=2,3, \ldots, 7$.

where $\Delta V_{n m}=V_{\mathrm{in}}^{*} V_{1 n}-V_{i m}^{*} V_{1 m}$. Figure 1 shows the total cross section for $p p \rightarrow Z^{\prime} \rightarrow Z\left(\rightarrow l^{+} l^{-}\right)+2 \tilde{\nu}_{1}(l=e, \mu)$, based on the diagrams (top panels) in Fig. 2 (summed and squared, thereby capturing the relative interference too), for different masses of the $Z^{\prime}$ and $\tilde{\nu}_{1}$ after satisfying all Higgs data constraints by using HiggsBounds [9] and HiggsSignals [10]. The scanned points have been generated over the following intervals of the BLSSM fundamental parameters: $10^{3} \mathrm{TeV} \leq M_{\tilde{l}}^{2} \leq 5 \times 10^{3} \mathrm{TeV},-500 \mathrm{TeV} \leq M_{\tilde{\nu}}^{2} \leq-10^{2} \mathrm{TeV}$, $10 \mathrm{TeV} \leq M_{\tilde{S}}^{2} \leq 50 \mathrm{TeV}, \quad 0.3 \leq g_{B-L} \leq 0.5, \quad-0.4 \leq \tilde{g} \leq$ $-0.2, \quad 4 \mathrm{TeV} \leq v_{1}^{\prime} \leq 6 \mathrm{TeV}$, and $3 \mathrm{TeV} \leq v_{2}^{\prime} \leq 5 \mathrm{TeV}$ plus, to ensure that the lightest $\tilde{\nu}_{1}$ is the LSP, we kept $M_{1}=M_{2}=M_{3}=6 \mathrm{TeV}$. A benchmark point will be chosen from the scanned ones to perform a detailed Monte Carlo analysis. As the latter will be based around $Z^{\prime}$ production and decay, we also have made sure that, on the one hand, the scan points do not fall out of the LEP (indirect) constraints and, on the other hand, the ensuing $Z^{\prime}$ will not have been discovered via LHC (direct) searches in the Drell-Yan (DY) mode already. We meet these conditions by adjusting the parameters of the chosen point as follows: $M_{Z^{\prime}}=2.9 \mathrm{TeV}, M_{\tilde{\nu}_{1}} \simeq 90 \mathrm{GeV}, g_{B-L}=0.5$, and $\tilde{g}=-0.25$.

\section{MONO-Z ANALYSIS}

In the following, we will develop an analysis aimed at extracting information about the lightest right-handed sneutrino of the BLSSM as the DM candidate through a dedicated mono- $Z$ search using a machine learning (ML) algorithm called "boosted decision tree" (BDT) [11,12]. The key to this approach is to rely on monojet evidence of DM in a kinematic regime compatible with $Z^{\prime}$ production and decay, ${ }^{1}$ so that, under a model dependent assumption (i.e., assuming the BLSSM), one can extract mono- $Z$ signatures leading to the identification of the DM properties, chiefly, of its spin. In fact, an intriguing feature of the mono- $Z$ analysis is the possible spin characterization of DM. Spin determination methods rely heavily on the final state spins and the chiral structure of the couplings. The two-body decays of neutralinos to a massive $Z$ boson and a DM neutralino produce a $Z$ boson in three helicity states, \pm 1 (transverse) and 0 (longitudinal). Reconstructing the three polarization states through the angular distributions of the $Z$ boson leptonic decays through $\tilde{\chi}_{i}^{0} \rightarrow \tilde{\chi}_{1}^{0} Z\left(\rightarrow l^{+} l^{-}\right)$ in the rest frame of the decaying $Z$ boson leads to a clear characterization of the spin state of the $Z$ boson. The angular distribution of the transverse states are $\propto$ $\left(1 \pm \cos ^{2} \theta\right)$ while the angular distribution of the longitudinal state is $\alpha \sin ^{2} \theta$, where $\theta$ is the angle between the lepton momentum direction and the $Z$ boson one in the latter rest frame. The decay width of the neutralino $\tilde{\chi}_{i}^{0}$ to transversely $(T)$ and longitudinally $(L)$ polarized $Z$ bosons is given by [16]. It is worth mentioning that the decay width of the longitudinal component of a $Z$ boson is suppressed with respect to its transverse ones [17].

\footnotetext{
${ }^{1}$ Contrary to Ref. [13], here, the contributions of the $Z$ and SMlike Higgs $\left(h_{\mathrm{SM}}\right)$ as mediators are very small due to a lower bound on the LSP mass; in particular, one has $M_{\tilde{\nu}_{1}}>M_{h_{\mathrm{SM}}} / 2>M_{Z} / 2$, in order to satisfy the invisible SM-like Higgs decay upper limit $[14,15]$. This means that $Z$ and $h_{\mathrm{SM}}$ propagators are off shell, unlike the $Z^{\prime}$ one. Further, the $Z^{\prime}$ couplings to sneutrinos are much stronger than those of the $Z$ and $h_{\mathrm{SM}}$. Finally, we will enforce a stiff MET cut to enhance the $Z^{\prime}$ component of the signal.
} 


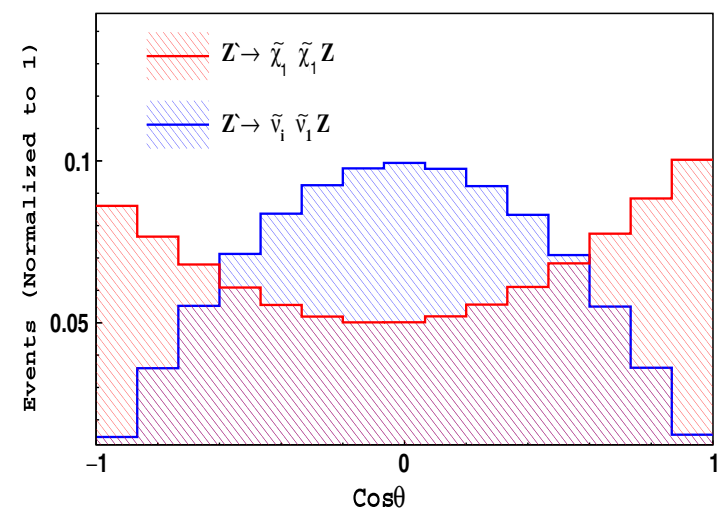

FIG. 3. Angular distribution of the final state lepton $(l=e, \mu)$ in the presence of a neutralino mediator in red (transverse polarization) and sneutrino mediator in blue (longitudinal polarization), where $\theta$ is the angle between the lepton and $Z$ boson directions in the $Z$ rest frame.

The two-body decays of heavier sneutrinos to a massive $Z$ boson and sneutrino DM, $\tilde{\nu}_{i} \rightarrow \tilde{\nu}_{1} Z\left(\rightarrow l^{+} l^{-}\right)$, produce a $Z$ boson in a zero-helicity (longitudinal) state only. This is because the helicity has to be conserved in the $S$-matrix and the fact that $\tilde{\nu}_{i}$ and $\tilde{\nu}_{1}$ are (pseudo)scalars forces the produced $Z$ boson to have a unique state (cf. Fig. 1 in [17]). Figure 3 shows the angular distribution of the final state lepton $l$ for $\tilde{\chi}_{i}^{0} \rightarrow \tilde{\chi}_{1}^{0} Z$ transitions in red and that for $\tilde{\nu}_{i} \rightarrow$ $\tilde{\nu}_{1} Z$ ones in blue. It is also worth noting that, in Refs. [1820], a similar approach based on angular distributions of leptonic $Z$ boson decays emerging from $\tilde{\chi}_{1}^{0} \rightarrow Z \tilde{G}$ transitions, with $\tilde{G}$ being the light gravitino, was considered to distinguish between a Higgsino-like and gauginolike neutralino in a model with gauge-mediated supersymmetry breaking (GMSB).

\section{RESULTS}

Given the Feynman diagrams underpinning mono- $Z$ production in the BLSSM case for sneutrino DM

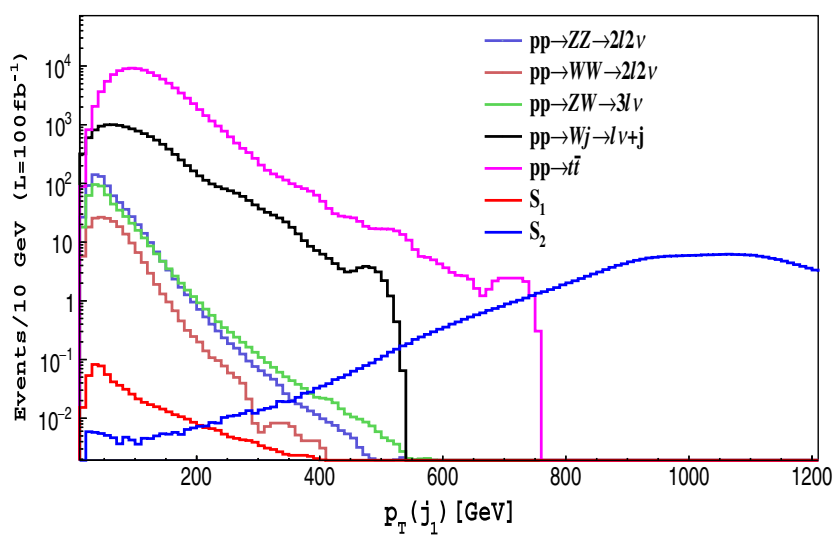

TABLE I. Total cross section in $\mathrm{pb}$ for the signal (split into the two topologies of Fig. 2, top panels) and the dominant background processes considered in our analysis. The samples have been produced with the following cuts: $p_{T}(l)>10 \mathrm{GeV}$, $p_{T}(j)>20 \mathrm{GeV}$, and $E_{T}>50 \mathrm{GeV}$.

\begin{tabular}{lcc}
\hline \hline & Process & $\sigma_{\text {tot }}(\mathrm{pb})$ \\
\hline$S_{1}$ & $p p \rightarrow Z^{\prime} Z\left(Z^{\prime} \rightarrow \tilde{\nu}_{1} \tilde{\nu}_{1}\right),(Z \rightarrow l l)$ & 0.0041 \\
$S_{2}$ & $p p \rightarrow Z^{\prime} \rightarrow \tilde{\nu}_{i} \tilde{\nu}_{1}\left(\tilde{\nu}_{i} \rightarrow \tilde{\nu}_{1} Z, Z \rightarrow l l\right)$ & 0.0115 \\
Backgrounds & $p p \rightarrow Z Z \rightarrow l l \nu \nu$ & 0.1256 \\
& $p p \rightarrow W W \rightarrow l l \nu \nu$ & 1.013 \\
& $p p \rightarrow Z W \rightarrow l l l \nu$ & 0.129 \\
& $p p \rightarrow W j \rightarrow l \nu+j$ & 2008 \\
& $p p \rightarrow t \bar{t}$ & 597 \\
\hline \hline
\end{tabular}

(see Fig. 2, top panels), the $Z$ boson decaying leptonically can be reconstructed as such by constraining the emerging electron and muon pairs to reproduce $M_{Z}$ within experimental dilepton mass resolution (we will not include $Z \rightarrow$ jet decays in the signal definition). The dominant irreducible background is $Z Z \rightarrow l^{+} l^{-} \bar{\nu} \nu$ and the other large noise in this category is $W^{+} W^{-} \rightarrow l^{+} \nu l^{-} \bar{\nu}$. As we reconstruct the $Z$ boson (specifically, by selecting the lepton pair that gives the closest value to the measured mass of the $Z$ boson), the reducible backgrounds must contain $Z \rightarrow l^{+} l^{-}$. Given the hadronic environment of the LHC, additional jet activity is possible. Hence, the final list of backgrounds in this category is as follows: $Z+$ jets, $Z Z \rightarrow l^{+} l^{-}+$jets, and $Z W \rightarrow l^{+} l^{-}+$jets. In addition, there are other reducible dilepton backgrounds with jets that we have dealt with: $t \bar{t} \rightarrow l^{+} \nu b l^{-} \bar{\nu} \bar{b}$, as well as $W^{ \pm}+$jets, which is reduced by a MET cut. The last quantitatively important background, purely leptonic, is $Z W \rightarrow l^{+} l^{-} l \nu$, with one electron misidentified as a jet. As preselection cuts we require $p_{T}(l)>10 \mathrm{GeV}, p_{T}(j)>20 \mathrm{GeV},|\eta(l / j)|<2.5$ (where $j$ represents any jet and $l$ any lepton), and $E_{T}>50 \mathrm{GeV}$. Table I shows the signal and background composition, wherein we emphasize the dominance of $S_{2}$ over $S_{1}$ owing

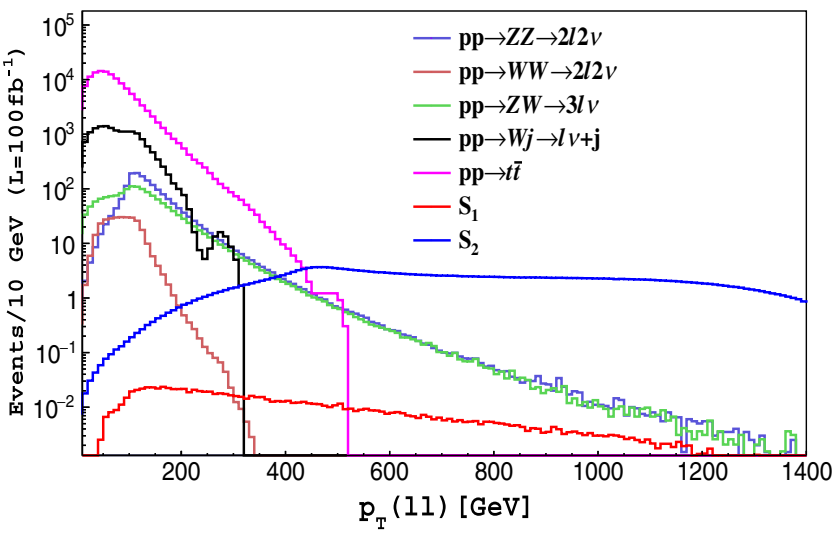

FIG. 4. Transverse momentum of the leading jet (left) and of the dilepton final state (right), with $S_{1}$ the signal process with $Z$ ISR (Fig. 2 left) and $S_{2}$ the signal process with $Z$ FSR (Fig. 2 right). 

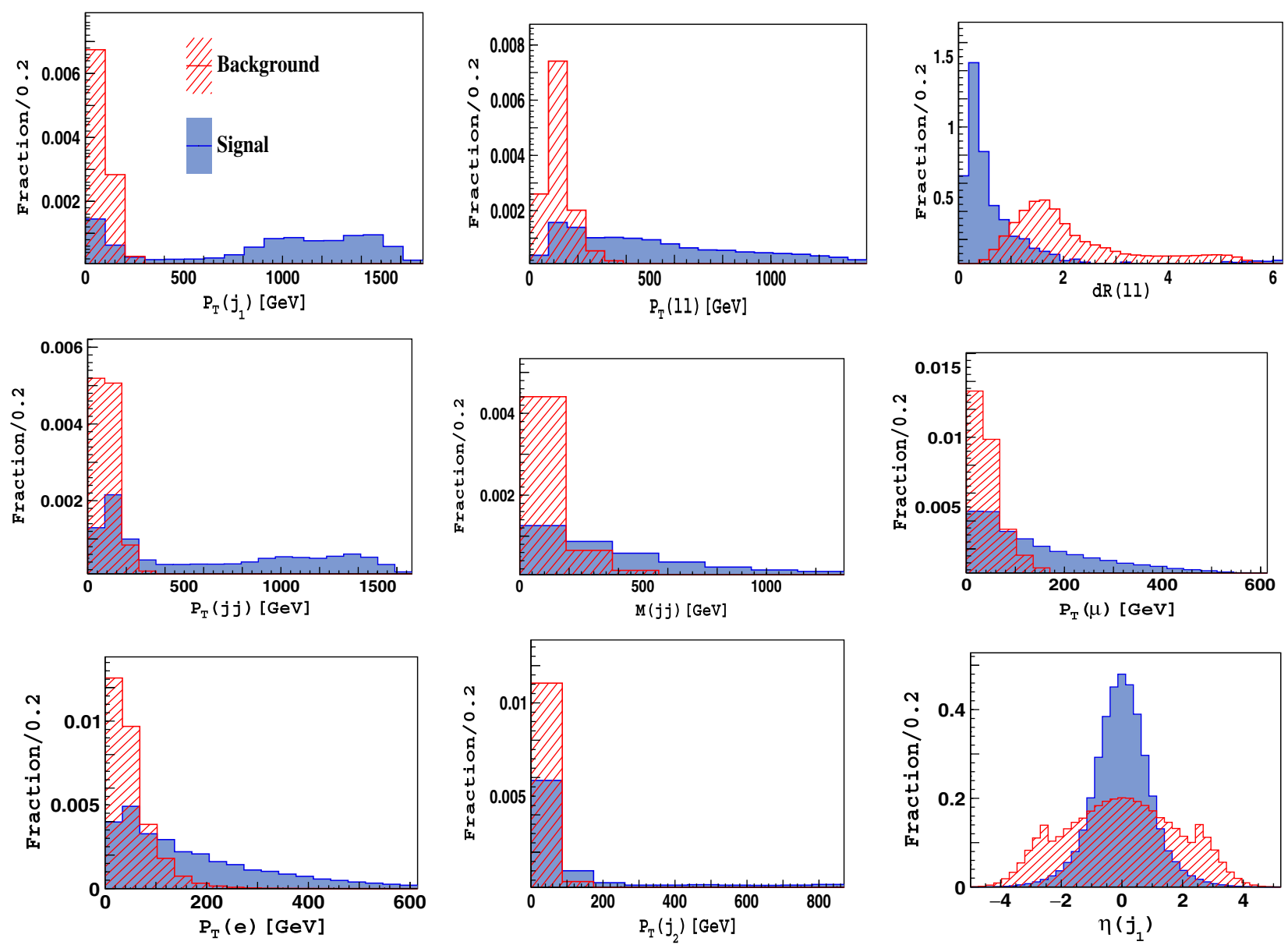

FIG. 5. Input distributions to the BDT for signal events in blue and all relevant backgrounds in red.

TABLE II. BDT ranking of the input variables in descending order of discriminative power.

\begin{tabular}{lcc}
\hline \hline Rank & Variable & Separation power \\
\hline 1 & $p_{T}\left(j_{1}\right)$ & $61.79 \%$ \\
2 & $p_{T}(l l)$ & $52.09 \%$ \\
3 & $\Delta R(l, l)$ & $48.35 \%$ \\
4 & $p_{T}(j j)$ & $43.81 \%$ \\
5 & $M(j j)$ & $40.90 \%$ \\
6 & $p_{T}(\mu)$ & $29.69 \%$ \\
7 & $p_{T}(e)$ & $29.66 \%$ \\
8 & $p_{T}\left(j_{2}\right)$ & $28.00 \%$ \\
9 & $\eta\left(j_{1}\right)$ & $18.44 \%$ \\
10 & $\eta(j j)$ & $8.173 \%$ \\
11 & $\Delta R\left(j j, l^{-}\right)$ & $6.434 \%$ \\
12 & $\Delta R\left(j j, l^{+}\right)$ & $6.129 \%$ \\
13 & $\eta(l l)$ & $6.112 \%$ \\
14 & $\eta\left(j_{2}\right)$ & $5.281 \%$ \\
15 & $\eta(\mu)$ & $5.189 \%$ \\
16 & $\Delta R\left(l l, j_{1}\right)$ & $5.002 \%$ \\
17 & $E_{T}$ & $4.928 \%$ \\
18 & $\Delta R\left(j_{1}, j_{2}\right)$ & $4.658 \%$ \\
19 & $\eta(e)$ & $4.571 \%$ \\
20 & $\Delta R\left(l l, j_{2}\right)$ & $4.358 \%$ \\
21 & $M(l l)$ & $2.713 \%$ \\
\hline \hline
\end{tabular}

to the $\tilde{\nu}_{i}$ multiplicity in the former, while the latter only sees the involvement of $\tilde{\nu}_{1}$. Moreover, we stress that, while the signal is mediated by a heavy gauge boson, $Z^{\prime}$, that leads to large MET, the whole background is not; thus we will eventually force the $E_{T}>100 \mathrm{GeV}$ condition into the BDT.

Upon enforcing all kinematic conditions above, relevant distributions are given as an input to our BDT in order to perform a multivariate analysis (MVA) [21]. An important feature of the MVA is that it can rank the input variables according to their ability to separate between signal and background events. For illustrative purposes, we show the first two variables ranked by the BDT for the signals $S_{1}$ and $S_{2}$ as well as all backgrounds separately in Fig. 4. Herein, a peculiar feature is the fact that the signal is mediated by a heavy $Z^{\prime}$, so that this causes the ISR jet in $S_{2}$ to recoil against a very massive object. Such kinematics pushes the transverse momentum distribution of the leading jet to be peaked around half of the $Z^{\prime}$ mass. This does not occur for $S_{1}$, though, owing to the presence of the ISR $Z$ also. For the dilepton transverse momentum, both signals have a much stiffer spectrum than any of the backgrounds, again, owing to $Z$ balancing the heavy $Z^{\prime}$ (in $S_{1}$ ) or else being ejected by the decay of the latter at large $p_{T}$ (in $S_{2}$ ). 

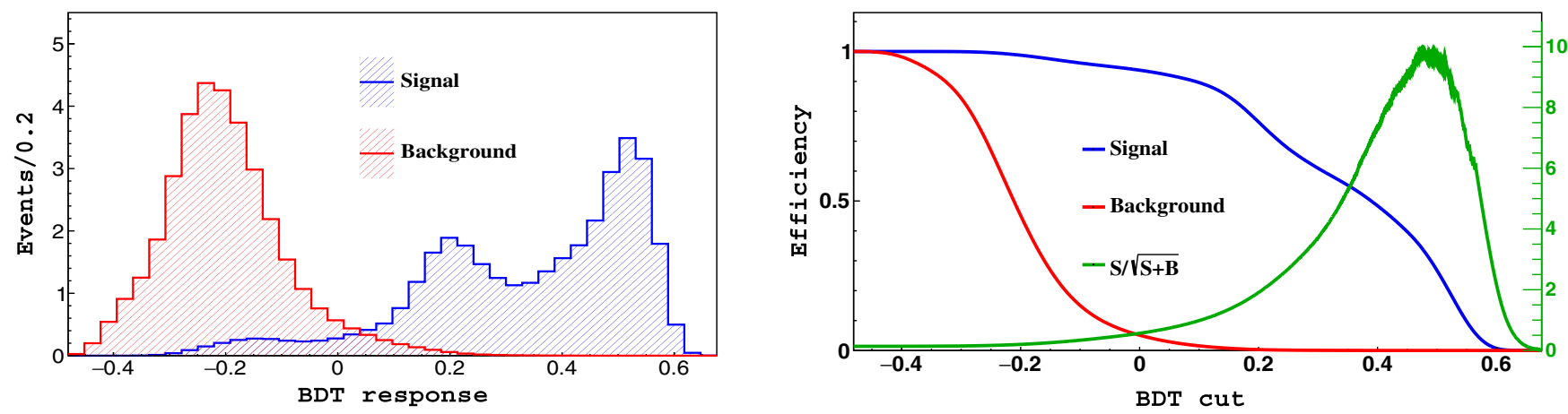

FIG. 6. Left: BDT response for the signal in blue and background in red. Right: BDT cut efficiency for signal in blue and background in red with the corresponding significance in green.

The discriminating power of the BDT relies on the fact that the signals and backgrounds may be characterized by different features that can be encoded into several distributions. For completeness, we sketch the first nine most important variables, as ranked by the BDT, in Fig. 5 (wherein backgrounds are shown cumulatively). Further, Table II shows the BDT ranking of all input variables according to their power in separating the signal and background events. Our ML approach is then based on a set of BDTs where each tree yields a binary output depending on whether an event is classified as signal-like or backgroundlike during the training session. The most important feature of the MVA algorithm is its possibility to combine the various discriminating kinematic distributions into one main discriminator, the BDT response, thus dealing with only one variable to maximize the signal rate over the background one. The BDT response ranges between -1 and +1 corresponding to pure background and pure signal, respectively.

After the aforementioned kinematic cuts (preselection), the total number of events for the signal is 656 while for the background the total is $2.3 \times 10^{7}$, both of which are passed to the MVA environment to perform the ML analysis. The resulting BDT response is shown in Fig. 6 (left) with signal events in blue and background ones in red. Enhancing the BDT cut efficiency is done by maximizing the function $S / \sqrt{S+B}$, where $S$ is the total signal rate and $B$ is the background one at the given luminosity. Hence, for the optimal value of the BDT cut set at 0.48 , the remaining signal events (222) and background ones (285) yield a significance of $9.8 \sigma$. This corresponds to a signal extraction efficiency of $34 \%$ and a background rejection efficiency of $1.2 \times 10^{-5}$. Figure 6 (right) shows the signal efficiency in blue and the background rejection efficiency in red versus the BDT cut with the corresponding significance in green.

Finally, notice that the analysis has been performed at a center-of-mass (CM) energy of $14 \mathrm{TeV}$ and integrated luminosity of $100 \mathrm{fb}^{-1}$. For the simulation of the signal and background event samples, we have used MadGraph5 (v2.4.3) [22]. Parton shower and hadronization have been carried out by PYTHIA6 [23,24] while a fast detector simulation by Delphes [25] was used.

\section{CONCLUSIONS}

We have shown that a ML based approach, as opposed to a standard cut-flow one, is well suited to extract a mono$Z\left(\rightarrow l^{+} l^{-}\right)$signal of the BLSSM at the LHC, with $14 \mathrm{TeV}$ and $100 \mathrm{fb}^{-1}$ of energy and luminosity, respectively. The latter is emerging from a heavy $Z^{\prime}$ boson decaying into sneutrinos, the lightest of which is the DM state of this scenario, eventually yielding a dilepton plus MET signature with additional jet activity. Furthermore, the ability of the $Z$ boson to couple directly to the DM state enables one to access the spin properties of the latter, specifically, by studying the angular behavior of either lepton relative to the $Z$ boson direction in its rest frame. We have illustrated this phenomenology using a single benchmark point in the BLSSM, compliant with current experimental limits. We defer to a future publication the illustration of such an approach applied to the entire BLSSM parameter space [26].

\section{ACKNOWLEDGMENTS}

W. A. acknowledges the support from the Department of Atomic Energy (DAE) "Neutrino Project" of the HarishChandra Research Institute (HRI). The work of A. H. is supported by the Swiss National Science Foundation. The work of S.K. and S.M. was partially supported by the H2020-MSCA-RISE-2014 Grant No. 645722 (NonMinimalHiggs). S. M. is financed in part through the NExT Institute. S. K. acknowledges partial support from the Durham IPPP Visiting Academics (DIVA) program. 
[1] S. Khalil and S. Moretti, The $B-L$ supersymmetric standard model with inverse seesaw at the large Hadron collider, Rep. Prog. Phys. 80, 036201 (2017).

[2] W. Abdallah and S. Khalil, Dark matter in $B-L$ supersymmetric standard model with inverse seesaw, J. Cosmol. Astropart. Phys. 04 (2017) 016.

[3] L. Delle Rose, S. Khalil, S. J. D. King, S. Kulkarni, C. Marzo, S. Moretti, and C.S. Un, Sneutrino dark matter in the BLSSM, J. High Energy Phys. 07 (2018) 100.

[4] L. Delle Rose, S. Khalil, S. J. D. King, C. Marzo, S. Moretti, and C.S. Un, Naturalness and dark matter in the supersymmetric $B-L$ extension of the standard model, Phys. Rev. D 96, 055004 (2017).

[5] W. Abdallah, J. Fiaschi, S. Khalil, and S. Moretti, Mono-jet, -photon and $-Z$ signals of a supersymmetric $(B-L)$ model at the Large Hadron Collider, J. High Energy Phys. 02 (2016) 157.

[6] W. Abdallah, A. Hammad, S. Khalil, and S. Moretti, Search for Mono-Higgs signals at the LHC in the $B-L$ supersymmetric Standard Model, Phys. Rev. D 95, 055019 (2017).

[7] S. Khalil, Radiative symmetry breaking in supersymmetric $B-L$ models with an inverse seesaw mechanism, Phys. Rev. D 94, 075003 (2016).

[8] S. Khalil, TeV-scale gauged $B-L$ symmetry with inverse seesaw mechanism, Phys. Rev. D 82, 077702 (2010).

[9] P. Bechtle, O. Brein, S. Heinemeyer, G. Weiglein, and K. E. Williams, HiggsBounds: Confronting arbitrary Higgs sectors with exclusion bounds from LEP and the tevatron, Comput. Phys. Commun. 181, 138 (2010).

[10] P. Bechtle, S. Heinemeyer, O. Stål, T. Stefaniak, and G. Weiglein, HiggsSignals: Confronting arbitrary Higgs sectors with measurements at the Tevatron and the LHC, Eur. Phys. J. C 74, 2711 (2014).

[11] A. Alves and K. Sinha, Searches for dark matter at the LHC: A multivariate analysis in the mono- $Z$ channel, Phys. Rev. D 92, 115013 (2015).

[12] A. Alves, A. C. O. Santos, and K. Sinha, Collider detection of dark matter electromagnetic anapole moments, Phys. Rev. D 97, 055023 (2018).

[13] E. Bernreuther, J. Horak, T. Plehn, and A. Butter, Actual physics behind mono-X, SciPost Phys. 5, 034 (2018).

[14] M. Aaboud et al. (ATLAS Collaboration), Search for invisible Higgs boson decays in vector boson fusion at $\sqrt{s}=13 \mathrm{TeV}$ with the ATLAS detector, Phys. Lett. B 793, 499 (2019).

[15] A. M. Sirunyan et al. (CMS Collaboration), Search for invisible decays of a Higgs boson produced through vector boson fusion in proton-proton collisions at $\sqrt{s}=13 \mathrm{TeV}$, Phys. Lett. B 793, 520 (2019).

[16] S. Y. Choi and Y. G. Kim, Analysis of the neutralino system in two body decays of neutralinos, Phys. Rev. D 69, 015011 (2004).

[17] S. Y. Choi, Z-boson polarisation as a model-discrimination analyzer, Phys. Rev. D 98, 115037 (2018).

[18] J. A. Bagger, K. T. Matchev, D. M. Pierce, and R.j. Zhang, Weak scale phenomenology in models with gauge mediated supersymmetry breaking, Phys. Rev. D 55, 3188 (1997).

[19] A. Ghosal, A. Kundu, and B. Mukhopadhyaya, Gauge mediated supersymmetry breaking signals in an electron photon collider, Phys. Rev. D 57, 1972 (1998).

[20] J. Dutta, B. Mukhopadhyaya, and S. K. Rai, Identifying Higgsino-like neutralino with a $\mathrm{keV}$-scale dark matter, arXiv:1904.08906.

[21] A. Hoecker, P. Speckmayer, J. Stelzer, J. Therhaag, E. von Toerne, and H. Voss, CERN Report No. CERNOPEN-2007-007, 2007.

[22] J. Alwall, R. Frederix, S. Frixione, V. Hirschi, F. Maltoni, O. Mattelaer, H.-S. Shao, T. Stelzer, P. Torrielli, and M. Zaro, The automated computation of tree-level and next-to-leading order differential cross sections, and their matching to parton shower simulations, J. High Energy Phys. 07 (2014) 079.

[23] T. Sjostrand, S. Mrenna, and P.Z. Skands, PYTHIA 6.4 physics and manual, J. High Energy Phys. 05 (2006) 026.

[24] T. Sjostrand, S. Mrenna, and P.Z. Skands, A brief introduction to PYTHIA 8.1, Comput. Phys. Commun. 178, 852 (2008).

[25] J. de Favereau, C. Delaere, P. Demin, A. Giammanco, V. Lemaître, A. Mertens, and M. Selvaggi (DELPHES 3 Collaboration), DELPHES 3, A modular framework for fast simulation of a generic collider experiment, J. High Energy Phys. 02 (2014) 057.

[26] W. Abdallah, A. Hammad, S. Khalil, and S. Moretti (to be published). 